\title{
Using Prenatal Blood Samples to Evaluate COVID-19 Rapid Serologic Tests Specificity
}

\author{
Jackeline Alger ${ }^{1,2,3,4} \cdot$ Maria Luisa Cafferata ${ }^{5,6} \cdot$ Tito Alvarado $^{1,3} \cdot$ Alvaro Ciganda $^{6}$ - Arturo Corrales A $^{1,7}$. \\ Hans Desale ${ }^{8}$. Arnaud Drouin ${ }^{9}$. Dahlene Fusco ${ }^{9}$. Jorge Garcia ${ }^{2,3}$. Luz Gibbons ${ }^{5}$. Emily Harville ${ }^{10}$. Wendy Lopez ${ }^{2,3}$. \\ Ivette Lorenzana $^{1,11}$. Fausto Muñoz-Lara ${ }^{1,12,13}$. Elsa Palou ${ }^{1,13}$. Eduardo Retes ${ }^{1,14}$. Manuel Sierra ${ }^{1,3,15,16}$. \\ Candela Stella $^{5} \cdot$ Xu Xiong $^{10} \cdot$ Lysien I. Zambrano $^{1,17} \cdot$ Pierre Buekens ${ }^{10,18}$
}

Published online: 16 July 2020

(c) Springer Science+Business Media, LLC, part of Springer Nature 2020

\begin{abstract}
Introduction Background cross-reactivity with other coronaviruses may reduce the specificity of COVID-19 rapid serologic tests. The vast majority of women attend prenatal care, which is a unique source of population-based blood samples appropriate for validation studies. We used stored 2018 serum samples from an existing pregnancy cohort study to evaluate the specificity of COVID-19 serologic rapid diagnostic tests.

Methods We randomly selected 120 stored serum samples from pregnant women enrolled in a cohort in 2018 in Tegucigalpa, Honduras, at least 1 year before the COVID-19 pandemic. We used stored serum to evaluate four lateral flow rapid diagnostic tests, following manufacturers' instructions. Pictures were taken for all tests and read by two blinded trained evaluators.

Results We evaluated 120, 80, 90, and 90 samples, respectively. Specificity for both IgM and IgG was $100 \%$ for the first two tests (95\% confidence intervals [CI] 97.0-100 and 95.5-100, respectively). The third test had a specificity of $98.9 \%(95 \%$ CI 94.0-100) for IgM and 94.4\% (95\% CI 87.5-98.2) for IgG. The fourth test had a specificity of $88.9 \%$ (95\% CI 80.5-94.5) for IgM and 100\% (95\% CI 96.0-100) for IgG.

Discussion COVID-19 serologic rapid tests are of variable specificity. Blood specimens from sentinel prenatal clinics provide an opportunity to validate serologic tests with population-based samples.
\end{abstract}

Keywords COVID-19 diagnostic testing $\cdot$ Pregnancy $\cdot$ Prenatal care $\cdot$ Serologic tests

\section{Significance}

What is already known on this subject? The validity of COVID-19 serologic rapid tests is not established, although they are becoming widely available. Sentinel prenatal clinics provide a unique opportunity to collect population-based blood samples for validation studies.

What this study adds? We used stored serum samples collected in a sentinel prenatal clinic before the COVID19 pandemic in Tegucigalpa, Honduras. We evaluated four rapid serologic tests and found specificities varying from 88.9 to $100 \%$. The high number of false positives for some tests would make them less suitable for field studies.

Pierre Buekens

pbuekens@tulane.edu

Extended author information available on the last page of the article

\section{Introduction}

There is an urgent need to obtain serologic data for SARSCoV-2, the virus causing COVID-19, for populations worldwide. Rapid diagnostic tests (RDTs) could play a key role in serologic surveys, but their validity is often not well documented for COVID-19 tests (Sethuraman et al. 2020). Blood routinely collected during prenatal care is a unique source of population-based samples which could be used to perform COVID-19 serologic studies (Buekens et al. 2020). UNICEF estimates that $87.4 \%$ of pregnant women in the world had at least one prenatal visit in 2014-2019 (UNICEF 2019). Data from sentinel prenatal clinics are used to generate population-based estimates of infectious diseases seroprevalence. For example, HIV/AIDS seroprevalence is often estimated from prenatal care data (Eaton et al. 2014). Additionally, prenatal care offers a unique opportunity to collect data from a population of both asymptomatic and 
symptomatic women. Most women receive prenatal care to some extent, providing a better estimate of population seroprevalence than samples based on blood banks, hospital biobanks, or volunteers.

The specificity of COVID-19 serologic tests has been questioned because of possible background cross-reactivity with other coronaviruses. This has been an issue for serological testing of SARS-CoV and MERS (Meyer et al. 2014). Specificity corresponds to the proportion of true negative among healthy subjects. We used stored serum samples collected before the pandemic from the Zika in Pregnancy in Honduras (ZIPH) cohort study to evaluate the specificity of COVID-19 serologic RDTs available for field studies in Honduras.

The first COVID-19 case in Honduras was reported on March 10, 2020 (Gobierno de Honduras 2020). Nine weeks later, the country has reported 2646 COVID-19 cases and a low testing rate of 572 tests per million inhabitants (Worldometer 2020). Soon after the start of the COVID-19 pandemic in Honduras, the government independent group named Plataforma Todos Contra el COVID-19 (all against COVID-19 platform) was formed. One of the working aims of this group has been to guide and scale up the laboratory testing for confirmation of individual cases and for epidemiologic surveillance studies (Plataforma Todos Contra el COVID-19 2020).

\section{Methods}

The current study leveraged stored serum samples from an existing prospective pregnancy cohort. The ZIPH study enrolls women at their first prenatal visit at the Alonso Suazo Health Center (Tegucigalpa, Honduras) and follows them up until delivery (Buekens et al. 2016). The health center is a large urban prenatal clinic with more than 1,100 new prenatal visits a year. We enrolled 3991 women from July 2016 to March 2020. Data management is centralized at the Institute for Clinical Effectiveness and Health Policy (Instituto de Efectividad Clínica y Sanitaria, IECS), Buenos Aires, Argentina.

The Data Center selected at random 120 women among 977 women who were enrolled in 2018 and had authorized their blood samples to be stored for 10 years for additional studies. All samples were collected more than 1 year before the first case of COVID-19 was reported in Honduras. The sample size was based on the number of RDTs available for evaluation. The Data Center prepared a list of study numbers (with control digits), which was emailed to Honduras where study labels were printed and pasted on data forms and on rapid tests immediately before use (Fig. 1). Data were collected on paper forms and entered in REDCap (Harris et al. 2009). Additionally, scans of each data form were taken and
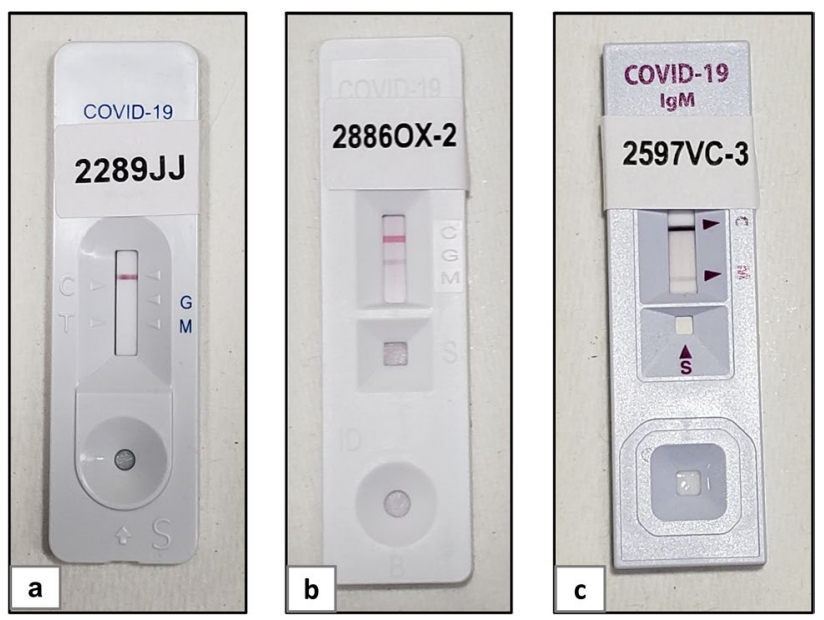

Fig. 1 a-c Digital picture of COVID-19 rapid serological test (RDT\#1) with negative result (a) and RDT\#3 with IgG (b) and RDT\#4 with IgM (c) positive results

were sent (encrypted) to IECS. This system allows for a digital backup of all study data forms.

Four lateral flow RDT were obtained by the Plataforma Todos Contra el COVID-19 through the Honduran Sanitary Regulatory Agency (Agencia Regulatoria Sanitaria, ARSA,) or directly as a donation. We used the following lateral flow RDTs:

RDT\#1: Hightop COVID-19 IgM/IgG Ab Rapid Test Kit (Qingdao Hantang Biological Technology Co., Ltd., Qingdao, China);

RDT\#2: COVID-19 IgG/IgM Rapid Test Kit (Nantong Egens Biotechnology Co. Ltd., Nantong, China);

RDT\#3: Orient Gene COVID-19 IgG/IgM Rapid Test (Zhejiang Orient Gene Biotech Co. Ltd, Huzhou, China).

RDT\#4: Standard Q COVID-19 IgM/IgG Duo Test (SD Biosensor, Gyeonggi-do, Republic of Korea).

Laboratory procedures were performed at the Departamento de Laboratorio Clínico, Hospital Escuela, Tegucigalpa, Honduras. One aliquot of frozen serum was thawed and kept at $4{ }^{\circ} \mathrm{C}$ for up to 3 days. Samples were brought to room temperature before testing. RDT packages were opened immediately before use. RDTs were performed following the manufacturers' instructions. Rapid tests were read at 10 to 20 min according to the respective RDT instructions, and digital pictures were immediately taken using a cell phone camera. Pictures were taken under constant lighting conditions using two light sources. We used a $3 \times$ magnification to allow the picture to be taken from enough distance to avoid shadows. The pictures were uploaded to an encrypted cloud site and were immediately available to the Data Center. The pictures were then uploaded to a secure website and were read by two blinded trained evaluators and compared to the initial reading in the laboratory. Discrepancies between the initial reading and readings from the blinded evaluators, or 
discrepancies between blinded evaluators, were resolved by an independent third blinded trained evaluator. This is similar to the approach we used earlier for Chagas RDTs (Buekens et al. 2013, 2018). We used OpenEpi version 3.01 to calculate $95 \%$ Fisher exact confidence intervals (CI).

This study was approved by Tulane University Institutional Review Board (893652; Amendment approval April 22, 2020), and by the Facultad de Ciencias Médicas, Universidad Nacional Autónoma de Honduras ethics committee (CEIB-079-2016; Amendment approval April 24, 2020). All women provided written informed consent at enrollment and authorized their blood samples to be stored for up to 10 years.

\section{Results}

Table 1 shows the characteristics of the selected women enrolled in the ZIPH cohort.

We tested 120 RDTs \#1, 80 RDTs \#2, and 90 RDTs \#3 and \#4 each. All tests had a clearly visible control band for all samples, except one for RDT\#4. Pictures were taken for all tests (Fig. 1). Both blinded evaluators agreed with the initial reading in 100\% (120/120) of RDT\#1 s, 96.3\% (77/80) of RDTs\#2, 95.6\% (86/90) of RDTs\#3, and 93.3\% (84/90) of RDT\#4. The third independent blinded evaluator made the final decision in 13 cases.

Table 2 shows that the specificity for both IgM and IgG was $100 \%$ for both RDT \#1 and RDT \#2, with 95\% CIs lower limits of $97.0 \%$ and $95.5 \%$, respectively. Specificity for RDT

Table 1 Maternal characteristics

\begin{tabular}{lll}
\hline & $\mathrm{N}=120$ & \\
\cline { 3 - 3 } & $\mathrm{n} / \mathrm{N}$ & $\%$ \\
\hline Maternal age & & \\
Between 15 and 19 & $31 / 120$ & 25.8 \\
Between 20 and 34 & $80 / 120$ & 66.7 \\
35 or higher & $9 / 120$ & 7.5 \\
Years of Education* & 9 & $(6-12)$ \\
Gestational age at first visit (weeks) & & \\
Between 1 and 13 & $69 / 93$ & 74.2 \\
Between 14 and 27 & $19 / 93$ & 20.4 \\
28 or higher & $5 / 93$ & 5.4 \\
Symptoms during pregnancy & & \\
Eruption & $0 / 116$ & 0.0 \\
Fever & $0 / 116$ & 0.0 \\
Arthralgia & $0 / 116$ & 0.0 \\
Arthritis & $0 / 116$ & 0.0 \\
Conjunctivitis & $0 / 116$ & 0.0 \\
At least one symptom & $0 / 116$ & 0.0 \\
\hline
\end{tabular}

*Median (interquartile range IQ)
Table 2 Serologic rapid diagnostic tests (RDTs): positive (+) tests for $\mathrm{IgM}$ and $\mathrm{IgG}$ and specificity (\% and $95 \%$ confidence intervals [CI])

\begin{tabular}{llllc}
\hline & IgM+ & $\begin{array}{l}\text { IgM specificity } \\
\%(95 \% \text { CI })\end{array}$ & IgG+ & $\begin{array}{l}\text { IgG specificity } \\
\%(95 \% \text { CI })\end{array}$ \\
\hline RDT \#1 & $0 / 120$ & $100(97.0-100)$ & $0 / 120$ & $100(97.0-100)$ \\
RDT \#2 & $0 / 80$ & $100(95.5-100)$ & $0 / 80$ & $100(95.5-100)$ \\
RDT \#3 & $1 / 90$ & $98.9(94.0-100)$ & $5 / 90$ & $94.4(87.5-98.2)$ \\
RDT \#4 & $10 / 90$ & $88.9(80.5-94.5)$ & $0 / 89$ & $100(96.0-100)$ \\
\hline
\end{tabular}

\#3 was $98.9 \%$ for $\operatorname{IgM}$ and $94.4 \%$ for $\operatorname{IgG}$. Specificity for RDT\#4 was $88.9 \%$ for $\operatorname{IgM}$ and $100 \%$ for $\operatorname{IgG}$ with a $95 \%$ CI lower limit of $96.0 \%$.

\section{Discussion}

Our results suggest that the COVID-19 serologic RDTs available in Honduras are of variable specificity when used in a general population. Specificity should have been $100 \%$ for all tests in these pre-COVID-19 samples. RDT \#1 and \#2 was $100 \%$ specific for both IgM and IgG, but specificity was as low as $88.9 \%$ for IgM for RDT\#4 and $94.4 \%$ for IgG for RDT \#3. None of the RDTs we tested are approved by the United States Food and Drug Administration (FDA), but all of them have a CE Mark for use in the European Union (Weissleder et al. 2020).

The specificities we found are comparable to the ones reported in the recent literature. A study of 10 serologic rapid tests found specificities varying from 91.6 to $100 \%$ among 108 blood donor plasma specimens collected in the United States before July 2018 (COVID-19 Testing Project 2020). A review of mostly unpublished data about nine serologic RDTs found specificities of $98.7 \%$ to $100 \%$, including RDT \#1, for which reported specificities were of $96.0 \%$ for IgM and $97.5 \%$ for IgG (Zainol Rashid et al. 2020). Hoffman et al. (2020) evaluated RDT \#3 and found higher specificities, $100 \%$ for IgM and $99.2 \%$ for IgG. A meta-analysis of regulatory data from serologic RDTs approved in Brazil found a pooled specificity of $97 \%$ for IgM and $98 \%$ for IgG (Castro et al. 2020). A study evaluating a RDT in China included 128 "clinical negative samples" and found one sample positive for $\operatorname{IgG}$ and 10 positive for $\operatorname{IgM}(\mathrm{Li}$ et al. 2020). Of note, "clinical negative samples" in Li. et al. (2020) were from unconfirmed suspected COVID-19 cases, which could explain the high frequency of positive IgM. Another study of a COVID-19 serologic RDT found no positive among 26 healthy blood donors, with a specificity of $100 \%$ (Shen et al. 2020).

Data from SARS-CoV and MERS suggested that background cross-reactivity with other human coronaviruses was an issue (Meyer et al. 2014). However, this was mostly the 
case for tests detecting antibodies against the whole virus rather than the ones detecting antibodies against specific recombinant antigens. More than $90 \%$ of adults have been reported to have antibodies against the four human coronaviruses (Gorse et al. 2010). Coronavirus infection among children with influenza-like illness occurs in Central America as in other countries of the Americas (Taylor et al. 2017). An unexpected finding from a study from Vietnam showed that human coronavirus infection was less frequent among children with influenza-like illness when housing was overcrowded (Nguyen et al. 2016). The 2012 Honduras Demographic and Health Survey (DHS) shows that in Tegucigalpa, $38 \%$ of the households have $>4$ people (Secretaría de Salud de Honduras, Instituto Nacional de Estadística e ICF International 2013). It is thus possible that circulation of other human coronaviruses is relatively low in our setting.

Rapid tests used for seroprevalence surveys need to be highly specific. The low specificity for IgG of RDT \#3 is a concern, as it would falsely classify more than $5 \%$ of those tested as positive. The other three RDTs were highly specific for IgG and would be recommended if further studies would show a high sensitivity. We conclude that among those tested, COVID-19 serologic RDTs specificity varies from a low 88.9 to $100 \%$ in unaffected populations from Tegucigalpa, Honduras. If further studies confirm their sensitivity in that setting, highly specific tests would be the instrument of choice for serological surveys. The validity of all RDTs should be carefully evaluated, and blood specimens from sentinel prenatal clinics provide an opportunity to test them with population-based samples.

Acknowledgements The Zika in Pregnancy in Honduras (ZIPH) study is partially funded by Vysnova Partners SC-2018-3045-TU. We thank the Honduras Agencia Regulatoria Sanitaria (ARSA) and Diagnósticos LIDSA, Honduras, for providing the rapid tests. We thank Van Tong, MPH, for her support to the ZIPH study. We thank Fernando Althabe, MD, for his support. We thank Andrea Meyer for editing this manuscript.

\section{References}

Buekens, P., Cafferata, M. L., Alger, J., Althabe, F., Belizán, J. M., Carlier, Y., et al. (2013). Congenital transmission of Trypanosoma cruzi in Argentina, Honduras, and Mexico: Study protocol. Reproductive Health, 10, 55.

Buekens, P., Alger, J., Althabe, F., Bergel, E., Berrueta, A. M., Bustillo, C., et al. (2016). Zika virus infection in pregnant women in Honduras: Study protocol. Reproductive Health, 13(1), 82.

Buekens, P., Cafferata, M. L., Alger, J., Althabe, F., Belizán, J. M., Bustamante, N., et al. (2018). Congenital transmission of Trypanosoma cruzi in Argentina, Honduras, and Mexico: An Observational Prospective Study. American Journal of Tropical Medicine and Hygiene, 98(2), 478-485.

Buekens, P., Alger, J., Bréart, G., Cafferata, M. L., Harville, E., \& Tomasso, G. (2020). A call for action for COVID-19 surveillance and research during pregnancy. The Lancet Global Health. https://doi.org/10.1016/S2214-109X(20)30206-0.

Castro, R., Luz, P. M., Wakimoto, M. D., Veloso, V. G., Grinsztejn, B., \& Perazzo, H. (2020). COVID-19: A meta-analysis of diagnostic test accuracy of commercial assays registered in Brazil. The Brazilian Journal of Infectious Diseases. https://doi. org/10.1016/j.bjid.2020.04.003.

COVID-19 Testing Project. (2020). Retrieved May 5, 2020, from https://covidtestingproject.org/.

Eaton, J. W., Rehle, T. M., Jooste, S., Nkambule, R., Kim, A. A., Mahy, M., et al. (2014). Recent HIV prevalence trends among pregnant women and all women in sub-Saharan Africa: Implications for HIV estimates. AIDS, 28(Suppl 4), S507-514.

Secretaría de Salud de Honduras, Instituto Nacional de Estadística e ICF International. (2013). Encuesta Nacional de Salud y Demografía 2011-2012. Tegucigalpa, Honduras: Secretaría de Salud de Honduras, Instituto Nacional de Estadística e ICF International.

Gobierno de Honduras. (2020). Coronavirus COVID-19 en Honduras. Retrieved May 7, 2020, from https://covid19honduras.org/.

Gorse, G. J., Patel, G. B., Vitale, J. N., \& O'Connor, T. Z. (2010). Prevalence of antibodies to four human coronaviruses is lower in nasal secretions than in serum. Clinical and Vaccine Immunology, 17(12), 1875-1880.

Harris, P. A., Taylor, R., Thielke, R., Payne, J., Gonzalez, N., \& Conde, J. G. (2009). Research electronic data capture (REDCap)-a metadata-driven methodology and workflow process for providing translational research informatics support. Journal of Biomedical Informatics, 42(2), 377-381.

Hoffman, T., Nissen, K., \& Krambrich, J., et al. (2020). Evaluation of a COVID-19 IgM and IgG rapid test; an efficient tool for assessment of past exposure to SARS-CoV-2. Infection Ecology \& Epidemiology, 10(1), 1754538. https://doi.org/10.1080/20008 686.2020 .1754538 .

Li, Z., Yi, Y., Luo, X., Xiong, N., Liu, Y., Li, S., et al. (2020). Development and clinical application of a rapid IgM-IgG combined antibody test for SARS-CoV-2 infection diagnosis. Journal of Medical Virology. https://doi.org/10.1002/jmv.25727.

Meyer, B., Drosten, C., \& Müller, M. A. (2014). Serological assays for emerging coronaviruses: Challenges and pitfalls. Virus Research, 194, 175-183.

Nguyen, D. N. T., Mai, L. Q., Bryant, J. E., Hang, N. L. K., Hoa, L. N. M., Nadjm, B., et al. (2016). Epidemiology and etiology of influenza-like-illness in households in Vietnam; It's not all about the kids! Journal of Clinical Virology, 82, 126-132.

Plataforma Todos Contra el COVID-19. (2020). Retrieved May 7, 2020, from https://www.bvs.hn/php/level.php?lang=es\&compo nent $=59$ \&item $=63$.

Sethuraman, N., Jeremiah, S. S., \& Ryo, A. (2020). Interpreting diagnostic tests for SARS-CoV-2. JAMA. https://doi.org/10.1001/ jama.2020.8259.

Shen, B., Zheng, Y., Zhang, X., Zhang, W., Wang, D., Jin, J., et al. (2020). Clinical evaluation of a rapid colloidal gold immunochromatography assay for SARS-Cov-2 IgM/IgG. American Journal of Translational Research, 12(4), 1348-1354.

Taylor, S., Lopez, P., Weckx, L., Borja-Tabora, C., Ulloa-Gutierrez, R., Lazcano-Ponce, E., et al. (2017). Respiratory viruses and influenza-like illness: Epidemiology and outcomes in children aged 6 months to 10 years in a multi-country population sample. Journal of Infection, 74(1), 29-41.

UNICEF. (2019). Antenatal care. Retrieved June 30, 2020, from https ://data.unicef.org/topic/maternal-health/antenatal-care/.

Weissleder, R., Lee, H., Ko, J., \& Pittet, M. (2020). COVID-19 diagnostics in context. Retrieved May 12, 2020, from https://csb. mgh.harvard.edu/covid. 
Worldometer. (2020). COVID-19 coronavirus pandemic. Retrieved May 7, 2020, from https://www.worldometers.info/coronaviru s/\#countries.

Zainol Rashid, Z., Othman, S. N., Abdul Samat, M. N., Ali, U. K., \& Wong, K. K. (2020). Diagnostic performance of COVID-19 serology assays. Malaysian Journal of Pathology, 42(1), 13-21.
Publisher's Note Springer Nature remains neutral with regard to jurisdictional claims in published maps and institutional affiliations.

\section{Affiliations}

\section{Jackeline Alger ${ }^{1,2,3,4} \cdot$ Maria Luisa Cafferata ${ }^{5,6}$. Tito Alvarado ${ }^{1,3} \cdot$ Alvaro Ciganda $^{6}$. Arturo Corrales A $^{1,7}$. Hans Desale ${ }^{8}$. Arnaud Drouin ${ }^{9}$. Dahlene Fusco ${ }^{9}$. Jorge Garcia ${ }^{2,3} \cdot$ Luz Gibbons $^{5}$. Emily Harville ${ }^{10}$. Wendy Lopez ${ }^{2,3}$. Ivette Lorenzana $^{1,11}$. Fausto Muñoz-Lara ${ }^{1,12,13}$. Elsa Palou ${ }^{1,13}$. Eduardo Retes ${ }^{1,14}$. Manuel Sierra 1,3,15,16. Candela Stella ${ }^{5} \cdot$ Xu Xiong $^{10} \cdot$ Lysien I. Zambrano ${ }^{1,17} \cdot$ Pierre Buekens ${ }^{10,18}$}

1 Plataforma Todos Contra el COVID-19, Tegucigalpa, Honduras

2 Departamento de Laboratorio Clínico, Hospital Escuela, Tegucigalpa, Honduras

3 Instituto de Enfermedades Infecciosas y Parasitología Antonio Vidal, Tegucigalpa, Honduras

4 Unidad de Investigación Científica, Facultad de Ciencias Médicas, Universidad Nacional Autónoma de Honduras (UNAH), Tegucigalpa, Honduras

5 Instituto de Efectividad Clínica y Sanitaria, Buenos Aires, Argentina

6 Unidad de Investigación Clínica y Epidemiológica, Montevideo, Uruguay

7 Ingeniería Gerencial, Tegucigalpa, Honduras

8 Department of Tropical Medicine, Tulane School of Public Health and Tropical Medicine, New Orleans, LA, USA

9 Department of Medicine, Tulane School of Medicine, New Orleans, LA, USA

10 Department of Epidemiology, Tulane School of Public Health and Tropical Medicine, New Orleans, LA, USA
11 Centro de Investigaciones Genéticas, Facultad de Ciencias, UNAH, Tegucigalpa, Honduras

12 Departamento de Medicina Interna, Hospital Escuela, Tegucigalpa, Honduras

13 Departamento de Medicina Interna, Facultad de Ciencias Médicas, UNAH, Tegucigalpa, Honduras

14 Dirección de Postgrado, Universidad Católica de Honduras Nuestra Señora Reina de La Paz, Tegucigalpa, Honduras

15 Facultad de Ciencias Médicas, UNAH, Tegucigalpa, Honduras

16 Facultad de Ciencias de la Salud, Universidad Tecnológica Centroamericana, Tegucigalpa, Honduras

17 Departamento de Ciencias Fisiológicas y Morfológicas, Facultad de Ciencias Médicas, UNAH, Tegucigalpa, Honduras

18 W. H. Watkins Professor of Epidemiology, School of Public Health and Tropical Medicine, Tulane University, 1440 Canal St., Suite 2001, New Orleans, LA 70112, USA 